\title{
Recidiva de tumor Phyllodes sarcomatoide en una mujer joven: a propósito de un caso
}

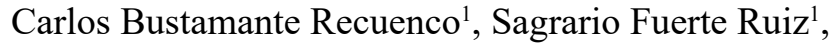 \\ Raquel León Ledesma ${ }^{1}$ y Eva María Lumbreras ${ }^{1}$
}

'Hospital Universitario de Getafe, Getafe, Madrid, España.

Recibido el 15 de octubre de 2018 y aceptado para publicación el 5 de diciembre de 2018

Correspondencia a:

Dr. Carlos Bustamante R. carlosb121990@gmail.com

\section{Recurrence of sarcomatoide phyllodes tumor in a young}

Introduction: Phyllodes tumor is a rare entity, with a high tendency to local recurrence and a malignancy rate of 5-10\%. The basis of its treatment is the surgical resection. Materials and Method: We report a 32-year-old woman who was diagnosed of a malignant Phyllodes tumor who had previously underwent conservative breast surgery and expander placement in another center. Two subsequent lumpectomies due to local recurrences were necessary. She was referred to our Hospital for she presented a new large-sized recurrence $(9.2 \mathrm{~cm})$ at the retroarelor area for assessment of new surgical treatment. Results: Surgical resection of the tumor (remnant of mammary tissue and pectoralis major muscle) and removal of the expander with immediate breast reconstruction with latissimus dorsi flap was performed. At the present time, the patient is pending radiotherapeutic treatment. Conclusions: Malignant Phyllodes tumor is a rare entity whose therapeutic management is based on margin- free surgical excision. Adjuvant chemoradiotherapy might be administered. It is very important to carry out a multidisciplinary and individualized management of each case to offer the best possible forecast.

Key words: Phyllodes; resection; recurrence.

\section{Resumen}

Introducción: El tumor Phyllodes mamario es una entidad muy poco frecuente, con tendencia a la recidiva local y una tasa de malignización de 5-10\%. La base de su tratamiento es la resección quirúrgica. Material y Métodos: Mujer de 32 años diagnosticada de un tumor Phyllodes maligno en mama derecha sometida en otro centro a cirugía conservadora con colocación de expansor y a dos tumorectomías posteriores por recidiva local. Fue referida a nuestro Hospital al presentar una nueva recidiva de gran tamaño $(9,2 \mathrm{~cm})$ para valoración de intervención quirúrgica. Resultados: Se realizó exéresis del tumor (remanente de tejido mamario y músculo pectoral mayor) y retirada del expansor con reconstrucción mamaria con colgajo de dorsal ancho en el mismo tiempo quirúrgico. En el momento actual la paciente se encuentra pendiente de iniciar tratamiento radioterápico. Conclusiones: El tumor Phyllodes maligno es una entidad poco frecuente y cuyo manejo terapéutico se basa en la resección quirúrgica con márgenes. De forma adyuvante se puede administrar quimiorradioterapia. Es de gran importancia realizar un manejo multidisciplinar e individualizado de cada caso para ofrecer el mejor pronóstico.

Palabras clave: Phyllodes; resección; recidiva.

\section{Introducción}

El tumor Phyllodes de mama es una entidad poco frecuente que se engloba dentro de los tumores fibroepiteliales y supone aproximadamente el 0,5$1 \%$ del total de tumores mamarios ${ }^{1}$. En la mayoría de los casos es una neoplasia de naturaleza benigna que aparece en mujeres en torno a la cuarta década de la vida. Se divide según sus características histológicas en tres categorías: benigna, borderline o maligna, siendo esta última forma particularmente excepcional, entre el 5-10\% del total. Sin embargo, su comportamiento clínico y su alta tasa de recidiva tras la resección local no guardan especial relación 
con su categoría histológica, siendo su evolución relativamente impredecible. Su pronóstico es bueno, al presentar hasta un $80 \%$ de supervivencia libre de enfermedad a los 5 años para las formas malignas tras recibir un tratamiento correcto ${ }^{2}$.

La resección quirúrgica es el tratamiento de elección del tumor Phyllodes. Al tratarse de una enfermedad poco frecuente las recomendaciones terapéuticas se basan en series de casos y estudios retrospectivos con escaso tamaño muestral, por lo que el grado de evidencia de las mismas es bajo en general. En el momento actual se recomienda la resección local amplia con márgenes de $1 \mathrm{~cm}$ o más para las formas benignas o borderline ${ }^{3}$ y la mastectomía simple si se trata de tumores malignos o recidivas ${ }^{2}$. Respecto al tratamiento adyuvante a realizar tras la resección quirúrgica también existe poca evidencia, por lo que no disponemos en el momento actual de un manejo sistematizado. En general, se puede recomendar la administración de radioterapia postoperatoria en las formas malignas y en los tumores recidivados. Sin embargo, el papel de la quimioterapia aún está por definir ${ }^{4}$.

Se comunica el caso de una mujer joven con varias cirugías previas por tumor phyllodes maligno en mama derecha que presentó una nueva recidiva de gran tamaño a nivel retroareolar. Dicha paciente fue referida a nuestro centro para valoración de nueva resección quirúrgica.

\section{Presentación del caso}

Paciente mujer de 32 años, intervenida en otro centro en varias ocasiones por tumor Phyllodes maligno de mama derecha. En primer lugar se realizó una tumorectomía, que tuvo lugar 4 años antes de la realización de este artículo. Debido al tamaño de la lesión extirpada fue necesario realizar una reconstrucción inmediata con expansor con simetrización de la mama contralateral. El resultado del análisis histológico fue de tumor Phyllodes de bajo grado de malignidad con márgenes quirúrgicos libres. Durante el seguimiento la paciente presentó dos recidivas locales al año y a los tres años que fueron tratadas quirúrgicamente mediante tumorectomía. En ambos casos fue necesaria una nueva intervención de ampliación de márgenes al ser positivos para malignidad. Se remitió a nuestro centro hace un año al presentar una nueva recidiva local a nivel retroareolar derecho para valoración de tratamiento quirúrgico conjunto por parte de los Servicios de Cirugía General y Digestiva y Cirugía Plástica.

A la exploración física la paciente presentaba eritema y edema generalizado en mama derecha, así como tumoración ulcerada de gran tamaño a nivel retroareloar (Figura 1). Se realizó ecografía y resonancia magnética ( $\mathrm{RM})$ mamaria, en las que se objetivó en mama derecha una masa tumoral de $9,2 \mathrm{~cm}$ con infiltración focal de $1 \mathrm{~cm}$ de la cápsula fibrosa del expansor colocado en cirugías previas y dudosa afectación en dicha área del pectoral mayor (Figura 2). También se realizó biopsia con aguja gruesa (BAG) del tumor, con resultado de tumor Phyllodes con sobrecrecimiento estromal y signos de agresividad histológica. Debido a los hallazgos clínico-radiológicos y a las numerosas recurrencias presentadas por la paciente, se decidió en la sesión multidisciplinar de patología mamaria realizar una nueva extirpación quirúrgica mediante mastectomía con retirada del expansor colocado previamente y una reconstrucción inmediata con colgajo de dorsal ancho.

Durante la intervención se realizó retirada del expansor y exéresis con márgenes del tumor, incluyendo el músculo pectoral mayor y la cápsula anterior del bolsillo subpectoral, al encontrarse ambas estructuras infiltradas macroscópicamente (Figura 3). No se realizó ningún tratamiento a nivel axilar al no existir evidencia de diseminación ganglionar.

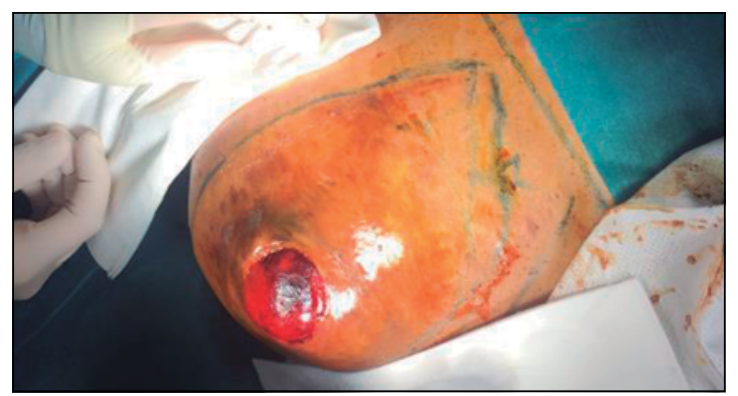

Figura 1. Hallazgo de tumor retroareolar de gran tamaño en exploración física.

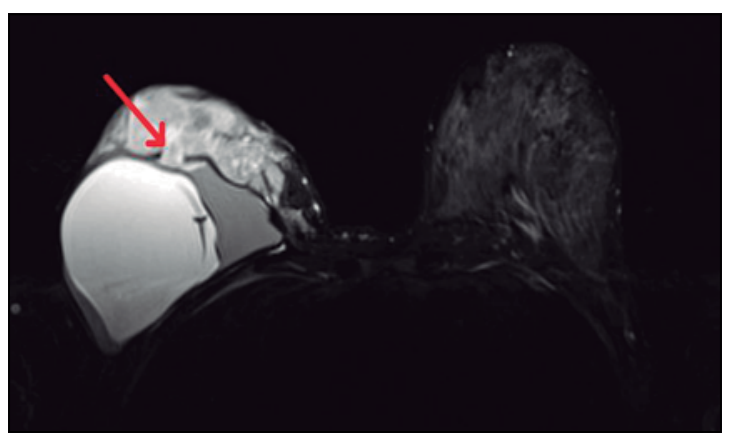

Figura 2. RM que muestra tumor Phyllodes retroareolar con infiltración de cápsula fibrosa y dudosa de músculo pectoral mayor (flecha roja). 


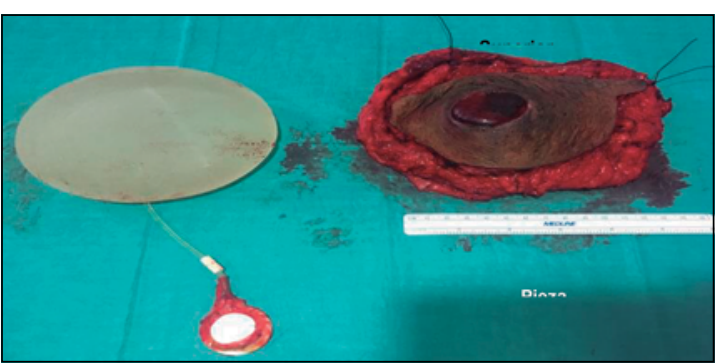

Figura 3. Pieza quirúrgica y expansor previo obtenidos tras la intervención.

Por parte del Servicio de Cirugía Plástica se realizó reconstrucción mamaria inmediata con colgajo de dorsal ancho (Figura 4). La paciente presentó buena evolución postoperatoria, siendo dada de alta con buen estado general. El análisis anatomopatológico de la pieza de resección mostró una tumoración de $9 \times 6 \times 4 \mathrm{~cm}$ con rotura de la fascia del pectoral mayor. Al análisis microscópico se observó un patrón fibroepitelial con un gran sobrecrecimiento estromal de carácter homólogo y una media de mitosis de $11 / 10$ por campo de gran aumento, catalogándose como un tumor Phyllodes maligno sarcomatoide. No se objetivó afectación de los márgenes quirúrgicos $(3,3 \mathrm{~cm}$ de margen libre a nivel lateral y $0,1 \mathrm{~cm} \mathrm{a}$ nivel posterior con la cápsula fibrosa de la prótesis mamaria).

La paciente fue remitida al Servicio de Oncología, cuyo personal decidió administrar radioterapia adyuvante, que se encuentra pendiente de recibir en el momento actual.

\section{Discusión}

El tumor Phyllodes es una entidad muy poco frecuente. Sus características epidemiológicas son poco conocidas, si bien se sabe que la edad media de aparición está descrita a los 45 años, aunque acontecen en un amplio rango de edades, desde los 10 hasta los 82 años $^{5}$. Son bilaterales en menos del $2,5 \%$ de los casos y pueden presentar un fibroadenoma concomitante hasta en un cuarto de los pacientes. No se conocen antecedentes familiares de riesgo, salvo su asociación con el síndrome de Li-Fraumeni ${ }^{6}$, que consiste en una predisposición a desarrollar un amplio rango de tumores (tumores de tejidos blandos, mamarios, leucemias y carcinomas adrenocorticales). Su principal característica es su alta tasa de recidiva local, que en las series asiáticas de mayor tamaño es de $10,9 \%, 14,4 \%$ y de $29,6 \%$

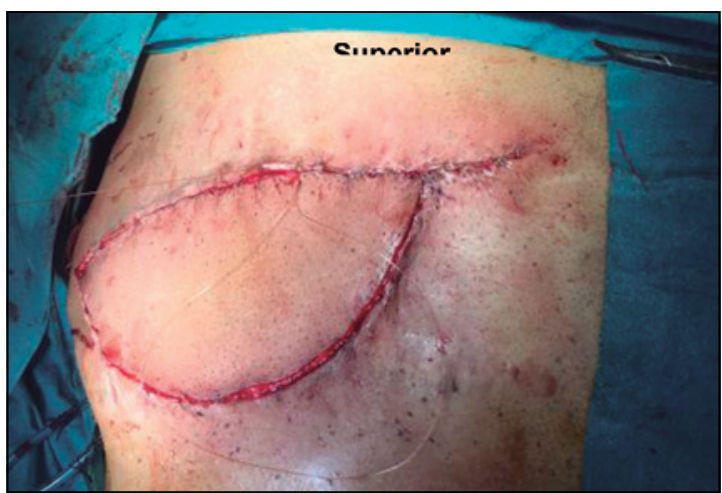

Figura 4. Resultado final tras reconstrucción.

para tumores benignos, borderline y malignos respectivamente 7 . Su diseminación se produce por vía hematógena (3-15\% de los casos), afectando principalmente al pulmón y a los huesos. Su pronóstico es bueno para las formas localizadas, si bien empeora drásticamente si se presentan metástasis en el momento del diagnóstico ${ }^{8}$.

Respecto a su presentación clínica, la mayoría se presentan como un nódulo mamario palpable, indoloro, firme y de crecimiento rápido, si bien en el $20 \%$ de los casos el tumor no es palpable. El tamaño medio es de $5 \mathrm{~cm}$ según la literatura ${ }^{9}$, si bien en la práctica clínica se pueden observar desde tumores de pocos centímetros hasta lesiones de gran tamaño. En todos los casos se debe realizar ecografía, mamografía y resonancia magnética para determinar con precisión la extensión local de la enfermedad. Sin embargo, ninguna de ellas tiene hallazgos patognomónicos, por lo que el diagnóstico de confirmación es anatomopatológico, debiéndose realizar una BAG de la lesión sospechosa en todos los casos, si bien a veces es necesaria su biopsia quirúrgica ${ }^{2}$. El diagnóstico diferencial se hará principalmente con el fibroadenoma en mujeres jóvenes o con el cáncer de mama en mujeres de mediana o de tercera edad.

En nuestra paciente el diagnóstico diferencial inicialmente se planteó entre una nueva recidiva o un carcinoma inflamatorio. Dados sus antecedentes personales y los hallazgos de las pruebas complementarias el diagnóstico final fue de recidiva de tumor Phyllodes maligno.

$\mathrm{Su}$ tratamiento es fundamentalmente quirúrgico. El objetivo principal debe ser la resección con márgenes libres, ya que su afectación tumoral es un factor de riesgo de recidiva local. En la revisión de Spilateri et al., se comunicó una tasa de recidiva de $31,8 \%$ si los márgenes eran positivos ${ }^{10}$. Respecto al efecto de la realización de una cirugía conservadora 
o radical, se ha demostrado que no influye sobre la tasa de recidiva local si los márgenes quirúrgicos son negativos ${ }^{11}$. Sin embargo, sí existen dudas en el momento actual sobre la amplitud que deben tener los mismos. La NCNN recomienda obtener unos márgenes libres de al menos $1 \mathrm{~cm}^{3}$, mientras que en ciertas revisiones, como la de Shaaban et al., se determina que no existen diferencias en la tasa de recurrencia entre dejar $1 \mathrm{~mm}$ o $1 \mathrm{~cm}^{12}$.

Respecto al tratamiento adyuvante, no existe consenso en el momento actual sobre las indicaciones de la quimio y radioterapia en esta patología. Este hecho se debe a su escasa incidencia y a la falta de estudios de calidad al respecto. Un 14,3\% del total de las pacientes intervenidas recibe radioterapia de forma adyuvante, siendo más frecuente su uso en pacientes sometidas a resección radical o con márgenes quirúrgicos menores de $1 \mathrm{~cm}^{13}$. El tratamiento radioterápico se ha relacionado con un mejor control de la recurrencia a nivel local (59 vs $86 \%$ ) independientemente del tipo de intervención realizada. Respecto al control de la diseminación a distancia, la evidencia sobre su utilidad es menor, si bien existen trabajos en los que se describe una disminución de la tasa de metástasis al recibir ratioterapia. En la revisión de Vithusa et al. ${ }^{14}$, realizada sobre 79 pacientes, se detectó una disminución de recidiva a distancia en las pacientes que recibieron radioterapia $(9,1 \%$ a 10 años) respecto a las que no la recibieron $(23 \%)$.

El papel de la quimioterapia, sin embargo, presenta una utilidad dudosa en esta patología. Únicamente existen estudios retrospectivos y series de casos, y en la mayoría de ellos no se obtiene beneficio en la supervivencia global ni libre de enfermedad ${ }^{15}$.

En nuestro caso, se decidió tratamiento adyuvante con radioterapia debido a las numerosas recurren- cias previas y al carácter maligno de la enfermedad. La administración de quimioterapia se desestimó dada la ausencia de evidencia sobre su beneficio.

\section{Conclusiones}

El tumor Phyllodes mamario continúa suponiendo un reto terapéutico al día de hoy. Su alta tasa de recidiva local y la falta de sistematización de su tratamiento suponen las principales cuestiones a superar.

La base del tratamiento es la resección quirúrgica con márgenes libres de enfermedad, realizando una cirugía conservadora siempre que sea posible para obtener un mejor resultado estético. La terapia adyuvante con radioterapia debe ser considerada en tumores malignos, recidivantes o con márgenes escasos para mejorar el control locorregional de la enfermedad.

Al tratarse de una enfermedad rara y de difícil tratamiento es de gran importancia la realización de un manejo multidisciplinar principalmente entre los servicios de Cirugía General, Cirugía Plástica y Oncología Médica para realizar el mejor tratamiento posible (oncológico y estético) de cada caso.

\section{Responsabilidades éticas}

Protección de personas y animales. Los autores declaran que para esta investigación no se han realizado experimentos en seres humanos ni en animales.

Confidencialidad de los datos. Los autores declaran que en este artículo no aparecen datos de pacientes.

Conflictos de interés: no hay.

\section{Bibliografía}

1. Geisler DP, Boyle MJ, Malnar KF, McGee JM, Nolen MC, Fortner SM, et al. Phyllodes tumors of the breast: a review of 32 cases. Am. Surgeon 2000;66:360-6.

2. Acea Nebril E, Aguayo Albasini JL, Basilio Bonet JE, Cansado Martínez P, Capote de Armas LF, Carrasco González $\mathrm{L}$, et al. Cirugía de la mama $1^{\circ} \mathrm{ed}$. Madrid: Arán Ediciones; 2006.

3. National Comprehensive Cancer Network. Phyllodes tumor (version 1.2016). 2016.
Available from: http://www.nccn.org/ professionals/physician_gls/pdf/ breast. pdf.

4. Tan Y, Acs G, Apple SK, Badve S, Bleiweiss IJ, Brogi E, et al. Phyllodes tumours of the breast: a consensus review. Histopathology 2016;68:5-21.

5. Parker SJ, Harries SA. Phyllodes tumours Postgrad Med J. 2001;77:428-35.

6. Birch JM, Alston RD, McNally RJ, Evans DG, Kelsey AM, Harris M, et al. Relative frequency and morphology of cancers in carriers of germline TP53 mutations. Oncogene 2011;20:4621-8.
7. Tan PH, Thike AA, Tan WJ, Thu MM, Busmanis I, Li H, et al. Predicting clinical behaviour of breast phyllodes tumours: a nomogram based on histological criteria and surgical margins. J Clin Pathol. 2012;65:69-76.

8. Lakhani SR, Ellis IO, Schnitt SJ, Tan $\mathrm{PH}$, van de Vijver MJ. World Health Organization Classification of Tumours of the Breast. Lyon: IARC Press; (2012).

9. Santos JS, García J, Bonal E, Martín R, Aparicio M. Tumor filodes de la mama: características clínicas, en imagen y anatomopatológicas a propósito de 18 


\section{CASOS CLÍNICOS}

casos. Rev Chil Radiol. 2007;13:90-7.

10. Spitaleri G, Toesca A, Botteri E, Bottiglieri L, Rotmensz N, Boselli S, et al. Breast phyllodes tumor: a review of literature and a single center retrospective series analysis. Crit Rev Oncol Hematol. 2013;88:427-36.

11. Tremblay-LeMay R, Hogue JC, Provencher L, Poirier B, Poirier E, Laberge S, et al. How wide should margins be for phyllodes tumors of the breast? Breast J. 2017;23: 315-22.
12. Shaaban M, Barthelmes L. Benign phyllodes tumours of the breast: (Over) treatment of margins - a literature review. Eur J Surg Oncol. 2017;43:1186-90.

13. Adesoye T, Neuman HB, Wilke LG, Schumacher JR, Steiman J, Greenberg $\mathrm{CC}$, et al. Current trends in the management of phyllodes tumors of the breast. Ann Surg Oncol. 2016;23:3199205.

14. Vithusha G, Dorst L, Lee J, Wan BA, Zhang L, Rakovitch E, et al. A retrospective review of phyllodes tumours of the breast: A single institution experience. The Breast 2018;38:52-7.

15. Morales-Vásquez J, González-Angulo AM, Broglio K, López- Basave HN, Gallardo D, Hortobagyi GN, et al. Adjuvant chemotherapy with doxorubicin anddacarbazine has no effect in recurrence-free survival of malignant phyllodes of the breast. Breast J. 2017;13:551-6. 